\title{
Fertilité après cancer du testicule
}

\author{
E. HUYGHE 1, P.F. THONNEAU 1,2 , P. PLANTE 1 \\ 1Service d'Urologie - Andrologie ; Hôpital La Grave ; 2Groupe de Recherche sur la fertilité humaine, \\ INSERM - Université Paul Sabatier ; Toulouse, France
}

\section{RESUME}

OBJECTIFS : Etudier la fertilité des patients atteins de cancer du testicule et analyser les facteurs prédictifs de la fertilité après traitements anti-cancéreux.

POPULATION ET METHODE : la série consécutive des 293 hommes suivis au CECOS Midi-Pyrénées entre 1978 et 1998 pour une tumeur germinale du testicule ont fait l'objet d'une analyse rétrospective à partir de leurs dossiers chirurgicaux, oncologiques et andrologiques, et ont été explorés du point de vue de la fertilité grâce à un questionnaire par courrier. Deux relances par courrier ont été réalisées, suivies d'un entretien téléphonique. Le taux de participation a été de $92 \%$.

RESULTATS : La fertilité après traitement de 277 hommes était connue : 138 d'entre eux avaient cherché à avoir une grossesse. 91 (66\%) ont eu une grossesse naturellement et $47(34 \%)$ n'y sont pas parvenu.

Les facteurs de mauvais pronostiques de la fertilité après traitement étaient : avoir un âge supérieur à 25 ans $(p<0.004)$, un antécédent de cryptorchidie $(p<0.01)$, une concentration de spermatozoïdes par $\mathrm{ml}$ avant traitement inférieure à 10 millions $(p<0.0001)$. Par contre il n'existait aucune relation significative entre la fertilité et le fait d'être traité par chimiothérapie $(p<0.6)$, ou radiothérapie $(p<0.6)$.

CONCLUSION : La population des hommes traités pour un cancer du testicule est à risque d'infertilité. Le fait d'être traité par radiothérapie ou chimiothérapie ne modifie pas la fertilité. Les facteurs prédictifs de la fertilité après traitement sont préexistants aux traitements.

Mots-clés : cancer du testicule, fertilité, chimiothérapie, radiothérapie

\section{INTRODUCTION}

Il y a beaucoup d'incertitude concernant la fertilité après traitement des hommes atteints d'un cancer du testicule, probablement par manque d'étude à large échelle sur leur devenir à long terme. Après les traitements radiothérapiques et chimiothérapiques utilisés dans le cadre du cancer du testicule, les patients sont initialement azoospermes ou oligospermes sévères $[1,3,9,12]$.

La question de leur fertilité après traitement est d'autant plus importante que ce cancer est la première cause de tumeur solide chez l'homme jeune, entre 20 et 34 ans [6], à un âge où il n'a pas encore eu d'enfant [14] et que ses chances de guérison dépassent actuellement $90 \%$. Conserver un potentiel de fertilité aux hommes soumis aux thérapeutiques stérilisantes est une des missions des CECOS en France. Depuis plus de vingt ans, la plupart des hommes atteints de cancers du testicule se sont vu proposer de réaliser une conservation de sperme avant le début des traitements anticancéreux afin d'utiliser les paillettes de sperme recueillies en cas d'infertilité post-thérapeutique.

Nous avons étudié la fertilité après traitement des hommes porteurs d'un cancer du testicule à partir de la population des patients venus réaliser une conservation de sperme au CECOS Midi-Pyrénées entre 1978 et 1999.

Correspondance :

P. Thonneau, Service d'Urologie - Andrologie ; Hôpital La Grave, Place Lange, 31052 Toulouse cedex thonneau.p@chu-toulouse.fr

Prix de DEA, XVII ${ }^{\circ}$ Congrès de la Société d'Andrologie de Langue Française, Bordeaux, 7-9 décembre 2000 


\section{PATIENTS ET METHODES}

Une série consécutive de 314 patients porteurs d'une tumeur du testicule ont été pris en charge au CECOS MidiPyrénées entre le 1 janvier 1978 et le 31 décembre 1998 afin de réaliser une conservation de sperme. Les patients dont l'histologie n'était pas une tumeur germinale, ont été exclus de l'étude. Finalement 293 patients ont été inclus dans l'étude.

Nous avons collecté les informations contenues dans leurs dossiers andrologiques, chirurgicaux et cancérologiques. Pour chaque patient, nous disposions d'informations concernant leurs caractéristiques socio-démographiques, leurs antécédents personnels et familiaux, l'anamnèse, le type de tumeur et le traitement institué, l'évolution de la maladie. Nous avions également connaissance de la valeur des principaux paramètres spermatiques lors de la conservation de sperme et au cours du suivi (pour les patients ayant consulté au CECOS Midi-Pyrénées après la fin des traitements).

Afin de connaître l'histoire reproductive des patients, un questionnaire à été élaboré par le Groupe de Recherche sur la Fertilité Humaine, à partir des données de la littérature.

Un pré-test a permis de s'assurer de son acceptation et de la compréhension des diverses questions. Après avoir testé l'option de collecter les informations par téléphone directement auprès des patients, nous avons préféré adresser le questionnaire par courrier, pour des raisons de confidentialité et d'efficacité liées à l'absence très fréquentes des coordonnées téléphoniques.

Le questionnaire standardisé était composé de deux parties : la fertilité avant et après les traitements anti-tumoraux.

La fertilité a été évaluée via le nombre total d'enfants (fausse-couche, IVG), la durée d'infécondité, les éventuels recours à des traitements de Procréation Médicalement Assistée, les malformations éventuelles. D'autres questions (dont les résultats ne seront pas présentés ici) concernaient le délai nécessaire pour concevoir, le délai entre la fin des traitements anti-tumoraux et la survenue d'une grossesse.

Le questionnaire a été adressé à tous les patients vivants accompagné d'une lettre d'information.

Nous avons procédé à 2 relances par courrier suivis d'une relance par téléphone, qui nous ont permis d'obtenir un taux de participation final de 91,6\%.

L'ensemble des informations collectées au niveau des dossiers de cancérologie, du CECOS et des chirurgiens, ainsi que les réponses au questionnaire ont été colligées sur un document synthétique qui a été saisi sur le logiciel Epi-Info.

L'analyse des données a été réalisée avec le logiciel STATA.
L'analyse statistique a comporté une étude descriptive des différentes variables. Les facteurs pronostics de la fertilité ont été testés par régression logistique et l'analyse de leur fertilité a fait appel aux modèles de Kaplan Meier et de Cox.

\section{RESULTATS}

Parmi les 293 patients présentant une tumeur germinale, 107 présentaient un séminome et 186 patients une tumeur germinale non séminomateuse.

$226 / 275(82 \%)$ patients ont été diagnostiqués à un stade inférieur à IIc selon la classification du Royal Marsden Hospital.

L'âge moyen ( \pm SD) des patients était de $28 \pm 6$ ans, allant de 15 à 52 ans. Il était de $26 \pm 6$ ans pour les patients atteints de tumeurs non séminomateuses, et de $31 \pm 6$ ans pour les séminomes.

$17 \%$ des patients présentaient un antécédent de cryptorchidie (dans 6 cas il existait une notion familiale de cryptorchidie).

Le spermogramme réalisé au moment de la conservation de sperme, a montré une numération moyenne de $27 \pm 33,2$ millions de spermatozoïdes par $\mathrm{ml}$, une mobilité de $36 \pm 20$ $\%$, une vitalité de $66 \pm 25 \%$ et un pourcentage de formes normales de $40 \pm 20 \%$. Il est à noter que $6 \%$ des patients étaient azoospermes, et que $22 \%$ d'entre eux avaient une numération inférieure à 10 millions de spermatozoïdes par $\mathrm{ml}$.

Dans $88 \%$ des cas, la chirurgie a consisté en une orchidectomie par voie inguinale.

101 patients ont reçu une radiothérapie, avec des doses totales s'étageant entre 2000 et 6500 rads. $70 / 89(79 \%)$ ont eu une irradiation sous-diaphragmatique, 19/89 (21\%) une irradiation sus et sous diaphragmatique. Pour 12 patients, le type de radiothérapie n'était pas connu.

La chimiothérapie a concerné 188 patients, dont 155 (82\%) ont reçu un protocole BEP, EP ou PVB, et 21 (11\%) une chimiothérapie plus agressive. Pour 12 patients, l'information n'était pas disponible.

24 patients ont eu une rechute qui a nécessité un traitement chimiothérapique et/ou radiothérapique complémentaire, et 8 ont présenté une atteinte controlatérale nécessitant une orchidectomie controlatérale.

Le taux de participation à l'enquête a été de $92 \%$ et le taux de perdus de vue de $4 \%$.

Au moment de la clôture de l'enquête (Août 2000), 17 patients étaient décédés, dont 11 de leur maladie. La mortalité pour les patients ayant au moins trois ans de suivi était de $2,7 \%$. 
Après traitement, la fertilité a pu être étudiée chez 277 patients, 91 avaient fait la preuve de leur fertilité (dont 83 naissances), 38 avaient cherché à avoir des enfants sans y parvenir, et $139(50 \%)$ n'avaient pas été exposés au risque de grossesse. Enfin 9 patients avaient eu un enfant grâce à une PMA.

Dans les analyses univariées, nous avons considéré d'un côté les patients ayant eu un événement reproductif « naturel » (c'est à dire sans recours à une PMA) $(n=91)$ et de l'autre les patients n'ayant pas réussi à obtenir une grossesse sans PMA: infécondité $(n=47)$.

Nous avons mis en évidence une relation significative entre l'infécondité et un âge au diagnostic supérieur à 25 ans $(\mathrm{p}=0,02)$, un antécédent de cryptorchidie $(\mathrm{p}=0,01)$, une numération de spermatozoïdes inférieure à 10 millions par millilitres au moment de la congélation $\left(\mathrm{p}<10^{-4}\right)$.

Nous n'avons pas mis en évidence de relation entre la fertilité après traitement et le fait $\mathrm{d}$ 'avoir eu un traitement de chimiothérapie ou de radiothérapie.

De même le fait d'avoir déjà apporté la preuve de sa fertilité avant les traitements ou le délai écoulé depuis la fin du traitement ne semblaient pas jouer de rôle significatif.

L'analyse multivariée confirme le rôle pronostic sur la fertilité après traitement de l'âge au moment du diagnostic, de l'antécédent de cryptorchidie, et de l'oligospermie avec une numération $<10 \mathrm{M} / \mathrm{ml}$ (Tableau 1).

\section{DISCUSSION}

La population des hommes atteints de tumeur du testicule est souvent présentée comme une population hypofertile avant tout traitement et qui reste à haut risque d'infertilité après traitement $[4,13]$. D'après les séries rapportées, environ la moitié de ces hommes ont une numération spermatique abaissée au moment du diagnostic de leur maladie [15].

Tableau 1 : Facteurs prédictifs de fertilité après cancer du testicule.

\begin{tabular}{|c|c|c|c|c|c|}
\hline \multirow[b]{2}{*}{ Tranche $25-35$ ans (réf. : tranche $<25$ ans) } & \multirow{2}{*}{$\begin{array}{l}\text { Odds Ratio } \\
4,9\end{array}$} & \multirow{2}{*}{$\begin{array}{l}\text { Ecart type } \\
2,7\end{array}$} & \multirow{2}{*}{$\begin{array}{l}P>|Z| \\
5.10^{-3}\end{array}$} & \multicolumn{2}{|c|}{ Intervalle de confiance $95 \%$} \\
\hline & & & & 1,6 & 14,7 \\
\hline Tranche $>35$ ans (réf. : tranche $<25$ ans) & 5,1 & 4,2 & 0,05 & 1,0 & 25,4 \\
\hline Antécédent de cryptorchidie & 3,7 & 2,0 & 0,02 & 1,3 & 10,5 \\
\hline Chimiothérapie & 2,0 & 1,2 & 0,3 & 0,6 & 6,4 \\
\hline Radiothérapie & 1,3 & 0,8 & 0,7 & 0,4 & 4,4 \\
\hline $\begin{array}{l}\text { N'a pas fait la preuve de sa fertilité avant } \\
\text { la maladie }\end{array}$ & 1,5 & 0,7 & 0,4 & 0,6 & 3,7 \\
\hline $\begin{array}{l}\text { Moins de } 10 \mathrm{M} / \mathrm{ml} \text { sur le spermogramme } \\
\text { de départ }\end{array}$ & 5,3 & 2,3 & $10^{-4}$ & 2,3 & 12,5 \\
\hline
\end{tabular}

Plusieurs études par questionnaire auprès des hommes traités pour un cancer du testicule ont porté sur leur fertilité après la fin des traitements $[2,8,10,11]$. Malgré des différences considérables de populations, de modalités de traitements et de questionnaires, leur analyse permet de dégager des convergences : Tout d'abord, environ la moitié des hommes interrogés n'ont pas été exposés au risque de grossesse depuis la fin de leurs traitements, malgré un recul par rapport aux traitements allant de 4 à 12 ans selon les études au moment du questionnaire (Huyghe : 139/277 soit 50\%, Arai : $50 / 85$ soit $42 \%$, Herr : $45 / 105$ soit $43 \%$, Hartmann : $40 / 98$ soit $65 \%$, Hansen : $117 / 158$ soit $74 \%$ ). L'étude de Hansen et coll. [8] trouve même un pourcentage d'hommes non exposés plus élevé ( $74 \%$ ), mais c'est également l'étude pour laquelle le recul par rapport aux traitements est le plus court (4 ans).

Ainsi, environ la moitié des effectifs inclus dans les études sur la fertilité après cancer du testicule ne sont pas concernés par l'étude, n'ayant pas souhaité avoir d'enfant depuis

Beaucoup d'hommes sont concernés par des changements dans leur vie de couple dans les années qui suivent le diagnostic du cancer. Arai et coll. [2] établirent un taux de divorce de $10 \%$. Dans notre étude, $44 \%$ des hommes ne vivaient pas en couple au moment de l'enquête.

De plus la période de diagnostic et de traitement du cancer du testicule est suivie par une période de difficulté psychologique marquée par le doute vis à vis de leur capacité à fonder une famille.

Selon les résultats d'une très intéressante enquête menée par Schover et coll. [14] sur un échantillon de 283 hommes chez lesquels un cancer avait été diagnostiqué avant l'âge de 35 ans, et estimés guéris de leur maladie, $30 \%$ avaient le sentiment de présenter une fertilité diminuée par rapport aux autres hommes. Gritz et coll. [7] rapportaient que $25 \%$ des hommes traités pour un cancer du testicule se trouvaient moins désirables. Dans l'étude de Arai et coll. [2], plus de $30 \%$ éprouvaient ce sentiment après l'orchidectomie. la fin des traitements. 
Parmi les hommes qui souhaitaient avoir un enfant, il semble que seulement 50-70\% y réussissent sans aide médicale à la procréation : Hartmann et coll. [10] ont mené une analyse rétrospective sur les dossiers de 124 patients. Il s'agissait de $80 \%$ de tumeurs germinales non séminomateuses, et surtout de tumeurs à un stade avancé ( $23 \%$ de stades I ). L'étude comportait l'envoi d'un questionnaire sur la fertilité auquel 98 patients ( $78 \%$ ) ont répondu. Parmi les 40 patients ayant souhaité avoir un enfant, 21 hommes $(53 \%)$ parvenaient à avoir un enfant après les traitements. Ce résultat est un peu plus faible que le notre $(66 \%)$. Ce résultat pourrait être expliqué par des différences de population (63\% de tumeurs germinales non séminomateuses et $56 \%$ de stades I dans notre étude). Par contre, nos résultats sont très voisins de ceux trouvés par Herr et coll. [11] . Ils étudièrent le devenir à long terme chez 105 patients présentant une tumeur germinale non séminomateuse du testicule de stade I, entrés dans un programme de surveillance entre 1979 et 1987. Les modalités de collecte des données étaient diverses : interrogatoire au cours des visites de contrôle, envoi d'un questionnaire courrier ou entretien téléphonique. Parmi les 63 couples qui ont essayé d'avoir des enfants sans facteur féminin d'infertilité, 41 , soit $65 \%$ eurent une grossesse.

Hansen et coll. [8] ont mené une étude par questionnaire chez 177 patients traités dans le Conté de Jutland du Nord au Danemark entre 1977 et 1986 pour une tumeur germinale du testicule. Les données concernant la fertilité après traitement sont connues chez 158 des 177 patients. Après traitement, 41 patients $(26 \%)$ avaient souhaité avoir un enfant. Parmi ceux-ci, 16 y sont parvenus. Les auteurs concluent que l'infertilité était encore un problème cinq ans après la fin des traitements chez $53 \%$ des patients. On peut remarquer la très grande proximité des résultats concernant le nombre de patients infertiles à 5 ans, observé dans l'étude de Hansen et coll. [8] (53\%), et dans la notre (54\%).

Ainsi, plus de la moitié des patients traités pour une tumeur testiculaire présentent une infertilité 5 ans après la fin de leurs traitements, et cette infertilité persiste au terme de l'étude chez un tiers des patients.

Les patients porteurs de cancer du testicule peuvent être traités par orchidectomie seule, mais le plus souvent un traitement complémentaire est administré (chimiothérapie, radiothérapie, ou curage ganglionnaire rétropéritonéal) selon le type et le stade de la tumeur.

La sensibilité du testicule aux effets de la radiothérapie et de la chimiothérapie est connue de longue date et les atteintes de la spermatogenèse entraînées par ces traitements bien établies $[1,13,5]$. Les patients traités par chimiothérapie ou radiothérapies sont donc considérés comme un groupe à haut risque de stérilité.

Cependant, la majorité des études ont porté sur les effets des traitements sur la qualité du sperme, et souvent selon des protocoles expérimentaux ou à haute dose, très éloignés des pratiques usuelles. Les études étudiant le lien entre la fertilité et les traitements usuels du cancer du testicule sont beaucoup moins nombreuses.

Nous n'avons pas identifié les traitements anticancéreux : radiothérapie comme chimiothérapie, comme des facteurs pronostiques de la fertilité (analyse univariée et multivarié). Hansen et coll. [8] ont obtenu les mêmes résultats : pas de différence de fécondité entre les hommes qui avaient reçu un traitement (chimiothérapique ou radiothérapique) et ceux qui n'en avaient pas reçu.

Arai et coll. [2], dans l'étude déjà citée ont obtenu des résultats différents : les traitements administrés ont été regroupés en 4 groupes : chimiothérapie $(n=15)$, chimiothérapie et curage ganglionnaire rétropéritonéal $(n=19)$, radiothérapie infradiaphragmatique $(n=42)$, surveillance $(\mathrm{n}=9)$. Parmi les patients désirant avoir des enfants, 3 naissances ont été obtenues chez les patients ayant eu une chimiothérapie, avec ou sans curage $(n=20)$, soit $15 \%$ de succès, 4 naissances chez les patients ayant eu une radiothérapie $(n=13)$, soit $31 \%$ de succès, et 4 naissances chez les patients ayant eu une surveillance, sans traitement complémentaire $(n=6)$, soit $67 \%$ de succès. La faiblesse des effectifs ne permet pas de tirer de conclusions à partir de ces résultats, en particulier dans le groupe Surveillance $(n=6)$.

Cependant, Arai et coll. [2] proposent d'offrir une conservation de sperme particulièrement aux patients devant recevoir un traitement cytotoxique (attitude qui ne paraît pas adoptée communément au Japon).

Herr et coll. [11] ont étudié la fertilité d'hommes inclus dans un protocole de surveillance après orchidectomie. Le principal événement survenant dans cette population était la survenue d'une évolution secondaire métastatique. Elle imposait la réalisation de traitements dont le détail n'est pas donné. La proportion d'hommes ayant eu un enfant est plus basse parmi les 27 patients ayant eu un traitement complémentaire pour une récidive de leur maladie (5 naissances), que dans le groupe des 78 hommes qui n'ont pas eu de traitement complémentaire (36 naissances). Cependant ces chiffres bruts sont difficilement interprétables sans connaître le nombre de couples qui souhaitaient réellement avoir des enfants et chez lesquels il n'y avait pas de facteur féminin d'infertilité : parmi ceux-ci, 35 grossesses ont été obtenues chez 43 patients n'ayant pas eu de récidive au cours du suivi ( $81 \%$ ) et 6 grossesses chez 20 patients ayant eu un traitement complémentaire $(30 \%)$. L'administration d'un traitement pour une récidive tumorale parait donc être un facteur d'infertilité chez ces patients. Il est vrai que la survenue d'une évolution métastatique, le plus souvent sous formes d'adénopathies métastatiques rétropéritonéales, indique un traitement associant une chimiothérapie et une chirurgie des masses résiduelle, souvent grevée de troubles de l'éjaculation. 
Nous avons observé que l'existence d'un antécédent de cryptorchidie et une oligospermie au moment de l'autoconservation étaient les principaux facteurs pronostiques de la fécondité après traitement. Ainsi, indépendamment de la maladie et de ses traitements, tout se passe comme si les patients à risque d'hypofécondité avant tout traitement conservaient un haut risque d'infécondité après traitement.

Parmi les hommes atteints de cancer du testicule qui n'ont pas encore eu d'enfant au moment du diagnostic de leur maladie, la majorité désireront devenir pères après le traitement de leur maladie (76\% d'après Schover et coll. [14]), aussi convient-il de prendre toutes les précautions afin de leur garantir un potentiel de fertilité après traitement.

L'identification de ces éléments «pronostiques » de la fertilité doit certainement inciter le clinicien à informer les patients de leur fertilité ultérieure, cela quels que soient les traitements proposée pour leur tumeur.

\section{CONCLUSION}

Les résultats de cette étude menée chez l'ensemble des patients atteints de tumeur du testicule ayant réalisé une conservation de sperme au CECOS Midi-Pyrénées au cours de ces vingt dernières années sont assez rassurants quant à la fertilité après traitement de ces patients. Par ailleurs, l'absence d'effet sur la fertilité des traitements de type chimiothérapie et/ou radiothérapie, illustre les capacités de récupération de la spermatogenèse.

\section{REFERENCES}

1. AMELAR R.D., DUBIN L., HOTCHKISS R.S. : Restoration of fertility following unilateral orchiectomy and radiation therapy for testicular tumors. J. Urol., 1971, 106, 714-718.

2. ARAI Y., KAWAKITA M., OKADA Y., YASHIDA 0. : Sexuality and fertility in long-term survivors of testicular cancer. J. Clin. Onco., 1997, 15, 1444-1448.

3. BERTHELSEN J.G. : Sperm counts and serum follicle stimulating hormone levels before and after radiotherapy and chemotherapy in men with testicular germ cell cancer. Fertil. Steril., 1984, 41, 281-285.

4. BERTHELSEN J.G., SKAKKEBAEK N.E. : Gonadal function in men with testis cancer. Fertil. Steril., 1983, 39, 68-74.

5. BOKEMEYER C., BERGER C.C., KUCZYK M.A., SCHMOLL H.J. : Evaluation of long-term toxicity after chemotherapy for testicular cancer. J. Clin. Oncol., 1996, 14, 2923-2932.

6. BOSL G.J., MOTZER R.J. : Medical progress: Testicular germ cell cancer. N. EngI. J. Med., 1997, 337, 242-253.

7. GRITZ E.R., WELLISCH D.K., WA NG H.D., et al : Long term effects of testicular cancer on sexual functioning in married couples. Cancer, 1989, 64, 1560-1567.

8. HANSEN P.V., GLAVIND K., PANDURO J., PEDERSEN M. Paternity in patients with testicular germ cell cancer : pretreatment and post-treatment findings. Eur. J. Cancer, 1991, 27, 13851389.

9. HANSEN S.W., BERTHELSEN J.G., VON DER MAASE H. : Long term fertility and Leydig cell function in patients treated for germ cell cancer with Cisplatin, Vinblastine and Bleomycin versus surveillance. J. Clin. Oncol., 1990, 8, 1695-1698.
12. 10. HARTMANN J.T., ALBRECHT C., SCHMOLL H.J., KUCZYK M.A., et al. : Long term effects on sexual function and fertility after treatment of testicular cancer. Br. J. Cancer, 1999, 80, 801-807.

11. HERR H.W. BAR CHAMA N., 0 SULLIVAN M., SOGANI P.C. : Paternity in men with stage I testis tumors on surveillance. J. Clin. Oncol., 1998, 16, 733-734.

12. JOHNSON D.H., HAINSWORTH J.D., LINDE R.B., GRECO F.A. : Testicular function following combination chemotherapy with Cisplatin. Vinblastine and Bleomycin. Medical and Pediatric Oncology, 1984, $12: 233-238$.

13. PETERSEN P.M., SKAKKEBAEK N.E., GIWERCMAN A. Gonadal function in men with testicular cancer : biological and clinical aspects. APMIS. 1998, 106, 24-36.

14. SCHOVER L.R., RYBICKI L.A., MARTIN B.A., BRINGELSEN K.A. : Having children after cancer : a pilot survey of survivors' attitudes and experiences. Cancer, 1999, 86, 697-709.

15. TUREK P.J., LOWTHER D.N., CARROLL P.R. : Fertility issues and their management in men with testis cancer. Urol. Clin. North. Am., 1998, 25, 517-531.

\section{ABSTRACT}

Fertility after testicular cancer

$$
\text { E. HUYGHE, P.F. THONNEAU, P. PLANTE }
$$

OBJECTIVES: To study the fertility of patients treated for testicular cancer and to identify predictive factors of infertility after treatment.

MATERIAL AND METHODS: 314 men with germ cell tumor, followed by the CECOS Midi-Pyrénées center between 1978 and 1998, were included in the study. They were evaluated retrospectively and interviewed by a mailed questionnaire concerning their reproductive history. If they failed to respond to the questionnaire, they were contacted twice by mail, and once by telephone. The response rate was $92 \%$.

RESULTS: The reproductive history of $\mathbf{2 7 7}$ men was known: 138 men had tried to have a child. $91(66 \%)$ succeeded and $47(34 \%)$ failed to achieve a "spontaneous" pregnancy. Age greater than 25 ( $p<0.004)$, a history of undescended testis, and a sperm count lower than 10 million per $\mathrm{ml}$ were inversely correlated with fertility $(p<0.004, p<0.01, p<0.0001$, respectively). However, no relationship was found between radiotherapy or chemotherapy and fertility after treatment.

CONCLUSION: Men treated for testicular cancer are at high risk of infertility. We identified various prognostic factors for fertility after treatment for testicular cancer: radiotherapy and chemotherapy had no significant effect on fertility.

Key words: testicular neoplasm, fertility, radiotherapy, chemotherapy. 